\title{
Novel Unsteady State Method of Measuring Permeability Tested on Fabric Materials
}

\author{
Anze Sitar* \\ Fondazione Bruno Kessler, Italy
}

The necessity of measuring permeability is encountered in number of applications and some of them have adequate measurement techniques available. However, in the fields of freeze drying, composite scaffolds, 3D printed materials, etc., the sample materials have unique shapes and sizes, which disables the use of standardized equipment. Hence, a novel method was developed to determine the permeability of a sample material by analyzing a high frequency unsteady state pressure measurement acquired during the permeation of a working fluid. The method is also suitable for in-line measurements due to the rapid acquisition and analysis. The developed method is novel, hence a comparison of the acquired results and the permeabilities measured with a standard device designed for measuring air permeability of fabric materials was made. Air was the permeating fluid in the novel unsteady state as well as in the referential steady state measurements. The measured permeabilities ranged from approximately $8 \mathrm{Da}$ to $50 \mathrm{Da}$ for the analyzed five fabric materials. The comparative analysis yielded an encouraging linear fit with a $R^{2}=0.98$ by including only one unsteady state measurement of each fabric sample. In addition, the presented method was capable of detecting the difference in permeability of the freeze-dried $5 \mathrm{wt} \%$ and $12 \mathrm{wt} \%$ aqueous mannitol solutions, which exhibit different permeabilities due to the different porosities after the same process of lyophilization. The possibility of determining permeabilities of freeze-dried pharmaceuticals is essential for lyophilization process optimization.

Keywords: novel measurement method; permeability; flow resistivity; unsteady state measurements; freeze-dried pharmaceuticals

Highlights

- Novel unsteady state method of determining permeability is proposed.

- Inexpensive, reliable and rapid acquisition of the experimental results is presented.

- $\quad$ The method is compared to standardized steady state measurements.

- Sample materials' size, shape and permeability is arbitrary.

- The first solution for determining permeability of freeze-dried pharmaceuticals is demonstrated.

\section{INTRODUCTION}

The technics of permeability determination are commonly divided into two categories: steady state and unsteady state methods. Both are most thoroughly studied in the field of geology due to the importance of rock permeability in the oil and gas industry. The two measurement categories are further divided into subcategories, which have unique features, recently summarized in Gao and Li [1]. The unsteady state methods have different variants with a common denominator. Namely, the measured signal is transient and the analysis of the experimental results requires a thorough theoretical knowledge, which was initially presented by Brace et al. [2] and afterwards modified by several authors [3] to [5]. Compared to the steady state experiments, the unsteady state measurements are more rapid, as shown by Zhong et al. [6], and more frequently applied for determining lower permeabilities, whereas the analysis of the results is generally much more complex and requires a profound theoretical background [7] as well as additional parameters: material and fluid compressibility, porosity, viscosity, etc. [8] and [9], which are often difficult to acquire.

Yong et al. [10] presented large discrepancies in liquid cross-plane permeability of glass fiber fabrics measured in 26 laboratories. However, the filter, membrane and fabric materials present no extraordinary challenges regarding the gas permeability measurements especially when determining the cross-plane steady state permeability as seen in [11].

Sheet materials are therefore ideal for verification of novel measurement methods, as there are several widely used and also standardized measurement methods available for determining the permeability. Most commonly used standardized equipment and procedures for measuring permeability are given in ASTM standards [12] and [13].

The currently available state of the art in steady or unsteady state methods of determining permeability is unsuitable to analyze non-extractable and arbitrarily shaped materials, which is the main advantage of the proposed technique. Hence, the use of this method is foreseen in the field of freeze-drying pharmaceuticals, as the product is not suitable for 
extraction and the permeability presents one of the most important parameters for designing the drying process. Currently used approaches of determining the porosity or permeability rely on Scanning Electron Microscopy [14] to [16]; X-ray microtomography [16] to [18] coupled with numerical simulations [19] and [20]; or solely on computational fluid dynamics [21] to [23]. Direct measurements of permeability in freeze dried products are non-existent, as the materials are brittle and therefore difficult to manipulate or extract from their containers, however the need to determine permeability is explicitly given in Fissore et al. [24] and Bobba et al. [25], as it would enable a more precise modelling and optimization of lyophilization cycles. The permeability is the most influential material property in the scope of lyophilization process, as the water vapor is required to permeate via sublimation through the material during the freeze drying process. The sublimation flow during drying is hindered by the materials flow resistance, therefore it is crucial to measure the product's permeability in order to evaluate and potentially improve the protocol of freeze drying.

The novel method allows for measuring the fluid permeability rate of sheet materials as well as other arbitrarily shaped materials. The method is flexible to perform measurements with different working fluids and various materials with a wide range of permeabilities. Additionally, the boundary and initial conditions of the experimental measurement can be adjusted to the expected process parameters to determine the permeability, which will affect the process of interest. Hence, the temperatures, pressures and volumetric flows during the experimental measurements can imitate the boundary conditions during freeze drying and therefore the intrinsic permeability is measured. To avoid elaborate physical modeling and acquisition of additional thermodynamic properties, a comparative analysis of the experimental results is recommended, which also diminishes the effect of fluid compressibility, as it is similar in all of the compared measurements. The rapid acquisition and analysis of the experimental results allow the use of the method in in-line quality control applications.

\section{EXPERIMENT AND THEORETICAL BACKGROUND}

\subsection{Experimental Setup and Procedure}

In order to validate the novel method of permeability measurement, reference steady state measurements were performed with a Mesdan Air Tronic instrument on five fabric materials with an area of $50 \mathrm{~cm}^{2}$ at a constant pressure difference of $100 \mathrm{~Pa}$. The permeability was calculated in accordance with the Darcy's law by using $1.84 \times 10^{-5} \mathrm{~Pa} \cdot \mathrm{s}$ as the dynamic viscosity of air at $25{ }^{\circ} \mathrm{C}$. The unsteady state method of determining permeability allows a prompt measurement performed in less than 50 ms and more importantly, it could also be used with arbitrarily shaped samples (for example: 3D printed materials, composite scaffolds, freeze dried materials, etc.). The unsteady state measurements of the fabric materials were performed with the experimental setup comprised from the components presented in Fig. 1. A computer and a data acquisition device are required for acquiring and analyzing the measured signal. The air supply chamber is connected with the upstream chamber through a valve to provide the required amount of the working fluid to the upstream chamber, which is equipped with a pressure signal to determine the initial pressure $p_{0}(t=0)$ as it is directly correlated with the quantity of the air in the chamber.

The pressure in the upstream chamber is higher compared to the pressure in the surroundings to ensure the driving force for the working fluid is available and

Computer

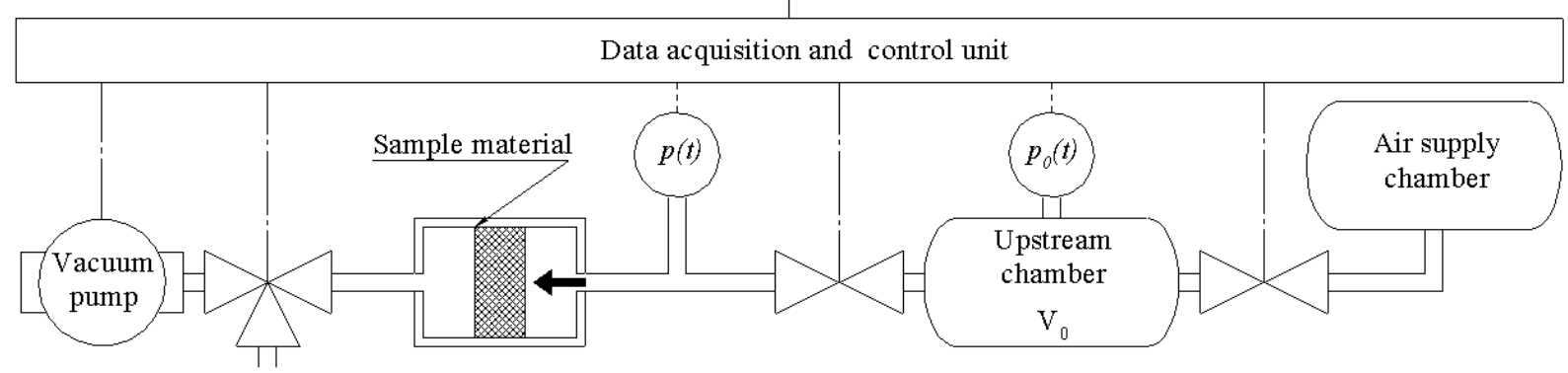

Fig. 1. Experimental setup for the unsteady state measurements 
properly oriented towards the left hand side in Fig. 1. The vacuum pump is installed at the outlet of the measured material in order to fully dry the sample material before the measurement of the permeability. After the desired amount of air is transferred to the upstream chamber, the valve connecting the supply and upstream chamber is closed and the measurements of both pressure signals $p(t)$ and $p_{0}(t)$ commence. At this moment both sides of the fabric material are subjected to air at the atmospheric pressure. Afterwards, the valve connecting the upstream chamber with the fabric material is opened and the air is allowed to dissipate through the fabric as the outlet side is in contact with the atmospheric pressure in the surroundings. The pressure sensor at the inlet side of the fabric material is used to acquire the pressure signal at a high frequency during the pulse of air permeating through the sample. The measured pressure signal $p(t)$ is in direct correlation with the sample's permeability.

\subsection{Data Reduction}

The permeability of a material in a viscous flow regime is defined with the Darcy's law, in which the permeability of the material $\kappa$ is determined with the equation

$$
\kappa=-\frac{\mu^{d V} / d t}{A^{d p} / d x} .
$$

The pressure difference across the material is $d p$ and the volumetric flow rate is $d V / d t$. The area and the thickness of the material are denoted with $A$ and $d x$, respectively, whereas the dynamic viscosity of the permeating fluid is $\mu$. The mass transfer resistance of the material is inversely proportional to the permeability in accordance with the equation

$$
R=\frac{d p}{d V / d t}=-\frac{\mu d x}{\kappa A} .
$$

The unsteady state measurements include the same volume of the permeating fluid $d V$, as it depends on the volume and the initial pressure in the upstream chamber, which were both constant during the experiments. Similarly, the viscosity of the fluid and the area of the sample were also kept constant during the transient measurements, whereas the thickness of the fabric samples varied from $0.135 \mathrm{~mm}$ to $0.5 \mathrm{~mm}$. The comparative analysis of the experimental results also allows us to exclude some of the otherwise influential parameters (for example: compressibility of air). These parameters become insignificant, as we are keeping them constant during the compared experimental runs. Therefore, in the established boundary conditions only the product of pressure difference and time $d p d t$ is the quantity required for comparative analysis of the materials' permeability. The tested fabric materials are subjected to a pulse of permeating air at the inlet side, whereas the fabric is in contact with atmospheric conditions at the outlet side. The unsteady pressure is measured at the inlet with an absolute pressure sensor, and the experimental results are afterwards normalized as stated with the equation

$$
p_{\text {norm }}(t)=\frac{p(t)-p(0)}{p_{\max }-p(0)} .
$$

The best results of analysis is expected at high speed measurements and large pressure differences, as the induced pressure pulse is rapidly declining when measuring highly permeable materials. The pressure signal $p(t)$ is measured at the inlet of the fabric material, whereas the pressures $p(0)$ and $p_{\max }$ are the initial pressure prior to the air pulse and the maximum possible value measured at the inlet. The maximum pressure value is defined as the pressure $p_{0}(0)$ initially set in the upstream chamber. The normalization of the pressure signal improves the comparability of the experimental results acquired in various measurements as there are potential differences (i) in the initial pressure in the upstream chamber $p_{\max }$ and (ii) in the initial pressure $p(0)$ at the inlet of the fabric, which is equal to the atmospheric pressure of the surroundings in the current experimental setup. Similar normalization procedure was used also for the time variable in order to improve the graphical distinctness of the differences between experimental results. The normalized time $t_{\text {norm }}$ is defined with the equation

$$
t_{\text {norm }}=\frac{t-t\left(p_{\max }\right)}{t_{\max }-t\left(p_{\max }\right)},
$$

in which the maximum time $t_{\max }$ was $70 \mathrm{~ms}$, defined as the time at which the pressure pulse is diminished back to the atmospheric pressure for all of the analyzed fabric materials. The value of $t\left(p_{\max }\right)$ was derived from the experimental results separately for every experimental run at the maximum achieved pressure at the inlet of the fabric material. The comparative parameter of the permeability can be defined as the maximum pressure, the slope of the pressure rise or decay, the area underneath the pressure signal in the $p(t)$ diagram or a combination of them all. 
The decision on the final comparative parameter depends on the initial and boundary conditions of the measurements and especially on the sample material's permeability. The performed measurements and analysis of the fabric materials exhibited the best results with comparing the following parameter

$$
P T_{\text {norm }}=\sum_{i} p_{\text {norm }}\left(t_{i}\right) \times \Delta t_{\text {norm }},
$$

which graphically presents the area underneath the normalized pressure curve in the $p_{\text {norm }}\left(t_{\text {norm }}\right)$ diagram.

\section{RESULTS AND DISCUSSION}

\subsection{Fabric Materials}

The unsteady state measurements were performed on five different fabric materials by utilizing the experimental setup presented in Fig. 1. Preliminary experiments were performed to adjust the experimental conditions. The initial pressure in the upstream chamber was varied from 0.1 bar to 0.5 bar, the number of layers of fabric materials ranged from 1 to 4 , whereas the upstream chamber volume and the measurement frequency remained constant. The results from the preliminary measurements showed a relatively high permeability of the fabric samples and consequentially low signal from the inlet pressure sensor, which is in viscous flow directly dependent on the following quantities

$$
d p=f(d x, 1 / A, 1 / \kappa, \mu, d V / d t) .
$$

Therefore, the pressure response of highly permeable fabric materials can be amended by varying their thickness or surface area perpendicular to the permeating flow, or by changing the volumetric flow, which is in our experimental setup indirectly controlled by the initial pressure in the upstream chamber. Hence, the decision on measuring 4 layers of fabric at the initial pressure of 0.5 bar in the upstream chamber was made to improve the measured pressure response. Additional rise of the initial upstream pressure, which would lower the noise to signal ratio was omitted in an attempt not to deviate even further from the pressures maintained in the referential steady state measurements, which were performed at a pressure difference of $100 \mathrm{~Pa}$. The pressure signal at the inlet was measured at a $4000 \mathrm{~Hz}$ frequency, which is presented without any filtering or conditioning in Fig. 2. All of the measurements performed on the four layers of fabric materials were set to have the maximum pressure signal at $25 \mathrm{~ms}$ and the pressure decayed to the initial value of the atmospheric pressure at $70 \mathrm{~ms}$. The inlet pressure sensor used was a an absolute pressure sensor, therefore some pressure differences in the initial states are observed due to the changes of the atmospheric pressure in the surroundings. This difference is negligible during the measurements on fabric materials 1,2 and 4, as all the experiments were performed on the same day with the same atmospheric pressure.

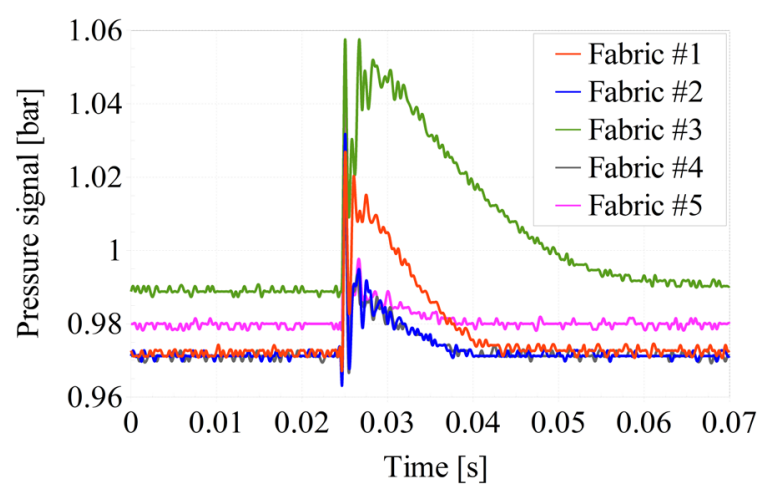

Fig. 2. Unconditioned pressure measured on five fabric materials vs. time

The pressure signals as well as time were normalized to further improve the comparability of the experimental results. The normalization was obtained in accordance with the Eqs. (3) and (4) and the corresponding results are depicted in Fig. 3. In addition, the analyzed data was reduced to only 141 pressure measurements for each fabric. The data was acquired at $4000 \mathrm{~Hz}$ from approximately $25 \mathrm{~ms}$ to 60 $\mathrm{ms}$, as shown in Fig. 2. The graphical representation of the measurements clearly shows some pressure oscillation, especially immediately after the maximum pressure is achieved. These oscillations were observed only in measurements of thin sheet materials at a relative large initial pressure in the upstream chamber, whereas experimental results of thicker less permeable samples or at lower pressure potentials hindered the pressure fluctuations. Therefore less distinct oscillations and a more smooth pressure pulse is achievable by adding additional layers of fabric materials, which inhibits the vibrations of the material during the permeating pulse of air. Sample materials' permeability could be compared on the basis of different experimental parameters (maximum pressure, slope of the pressure signal, etc.), however the relatively high permeabilities of the analyzed fabric materials imposed the comparison of the area below the graphs presented in Fig. 3, which was calculated in accordance with the Eq. (5) as the 
$P T_{\text {norm }}$. The final compared parameter included the pressure signal in the range of $0 \leq t_{\text {norm }} \leq 0.78$, which corresponds to the absolute time span of $25 \mathrm{~ms} \leq t \leq$ $60 \mathrm{~ms}$.

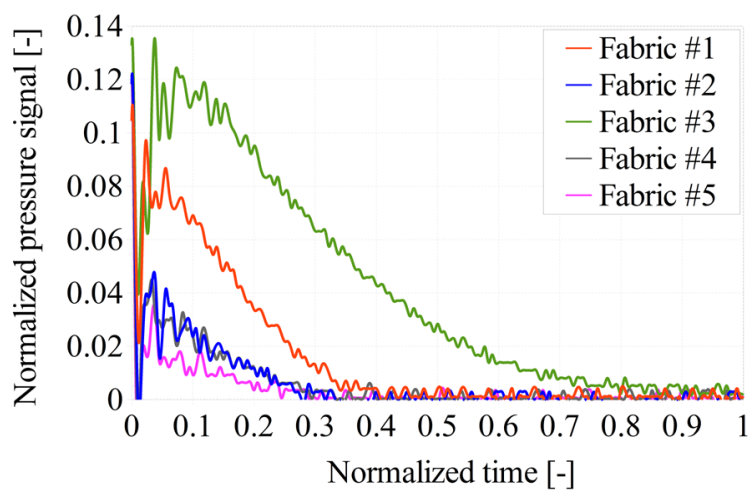

Fig. 3. Normalized pressure measured on five fabric materials vs. normalized time

The comparison of the unsteady state measured parameter $d x / P T_{\text {norm }}$ and the permeability $\kappa$ measured at a steady state is presented in Fig. 4. The compared parameter is the inverse value of $P T_{\text {norm }}$ multiplied with the sample thickness $d x$ as this combined value is theoretically proportional to the permeability. The presented analysis shows proportional changes in the unsteady state measurements of all five fabric materials compared to the referential steady state permeability. The comparison of the unsteady state parameter and referential permeabilities is especially encouraging as: (i) the experimental conditions and pressure sensors could be further optimized; (ii) the measured results are not conditioned in any way; (iii) only one measurement was performed for each sample material; (iv) utterly different steady and unsteady state measurement protocols provide completely comparable experimental results.

The complete set of the experimental results are presented in Table 1. The reference steady state measurements for all five fabric materials were performed by measuring the volumetric flow at a constant pressure difference across the sample. The reference permeabilities were calculated with the Darcy's law and range from approximately 8 Da to 50 Da for the analyzed fabrics.

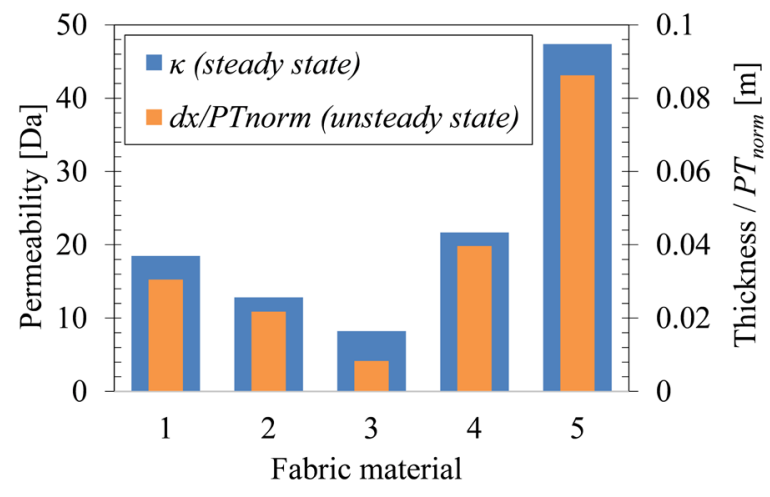

Fig. 4. Comparison of steady and unsteady state measurements

The unsteady state permeability comparison parameter was calculated from the normalized pressure signal and normalized time step. The steady state permeability in comparison with the unsteady state permeability parameter is presented in Fig. 5 with an addition of a linear curve fit given in Eq. (7). The unsteady state permeability derived from the linear fit as well as the relative difference compared to the reference permeability are also included in Table 1.

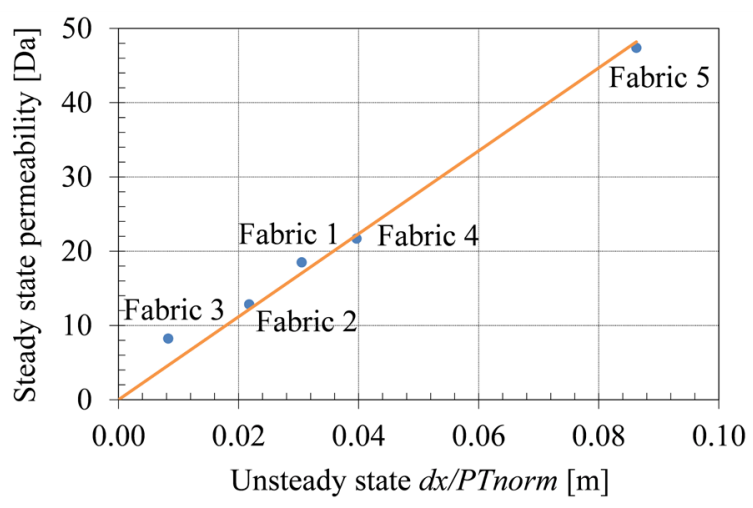

Fig. 5. Linear interpolation of the experimental results

Table 1. Summary of the experimental results and calculations

\begin{tabular}{|c|c|c|c|c|c|c|}
\hline & \multirow[b]{2}{*}{$\begin{array}{l}\text { Fabric thickness } \\
{[\mathrm{mm}]}\end{array}$} & \multicolumn{2}{|c|}{ Steady state measurements } & \multicolumn{2}{|c|}{ Unsteady state measurements } & \multirow[b]{2}{*}{$\begin{array}{c}\text { Relative difference } \\
{[\%]}\end{array}$} \\
\hline & & $\begin{array}{c}\text { Volumetric flow } \\
{[1 / \mathrm{min}]}\end{array}$ & $\begin{array}{c}\text { Permeability } \\
\text { [Da] }\end{array}$ & $\begin{array}{l}\text { Unsteady state } \\
d x / P T_{\text {norm }}[\mathrm{m}]\end{array}$ & $\begin{array}{c}\text { Calculated } \\
\text { permeability [Da] }\end{array}$ & \\
\hline Fabric 1 & 0.500 & 59.6 & 18.49 & 0.031 & 17.05 & -7.8 \\
\hline Fabric 2 & 0.135 & 153.2 & 12.83 & 0.022 & 12.16 & -5.2 \\
\hline Fabric 3 & 0.350 & 37.9 & 8.23 & 0.008 & 4.63 & -43.8 \\
\hline Fabric 4 & 0.250 & 139.8 & 21.68 & 0.040 & 22.15 & 2.2 \\
\hline Fabric 5 & 0.330 & 231.4 & 47.37 & 0.086 & 48.18 & 1.7 \\
\hline
\end{tabular}


The relative difference is the largest at the lowest permeability, which is anticipated, whereas the absolute differences are more comparable ranging from $-3.60 \mathrm{Da}$ to $+0.81 \mathrm{Da}$. The unsteady state method could be further improved by optimization of measurement equipment, operating conditions and acquiring a vast number of measurement results. The latter is achieved with ease, as each measurement required less than $50 \mathrm{~ms}$ to conclude.

The simple linear regression of the experimental results yielded the following equation with the coefficient of determination calculated at $R^{2}=0.98$

$$
\kappa=558.5 \times d x / P T_{\text {norm }} .
$$

The trend line of the referential steady state and unsteady state measurements was linear in the range of the results. The experimental data can be fitted with an arbitrary form of the fitting curve, if the experimental results would deviate from the linear trend. The current linear form of the regression in Eq. (7) has the coefficient 558.5 with a unit of $\mathrm{Da} / \mathrm{m}$ multiplied with $d x / P T_{\text {norm }}$ in meters, hence the calculated permeability is in $D a$. The presented regression model included all of the unsteady state measurements, as the objective was to test the novel measurement method, which was successful due to the promising trends achieved with a limited amount of measurements. The calculated trend line equation can be used without additional referential steady state measurements as long as the measured sample's permeability ranges from 8 Da to $50 \mathrm{Da}$. Any extrapolation of the linear equation, Eq. (7), is not appropriate, as we are relying on the comparative approach. Otherwise additional referential measurements are required to derive a renewed trend line valid in the broadened range of permeabilities.

\subsection{Freeze-dried Pharmaceuticals}

After the measuring method was evaluated on fabric materials, the transition to measuring freeze-dried materials in vials was made. The main challenges of measuring the lyophilized material is the brittleness of the material, which prohibits its' extraction from the vial. Hence, all of the measurements have to be made in-situ. WE have tested three different vials: (1) empty vial, (2) vial with a freeze-dried $5 \%$ aqueous solution of mannitol, and (3) a vial with a freeze dried $12 \%$ aqueous solution of mannitol. The inlet and outlet to the vial were both made through the rubber cap of the vial as seen in Fig. 6. The initial amount of the solution in the vial was $4 \mathrm{ml}$, which occupied approximately
$1 / 3$ of the vial volume, as it is commonly seen in lyophilized pharmaceuticals.

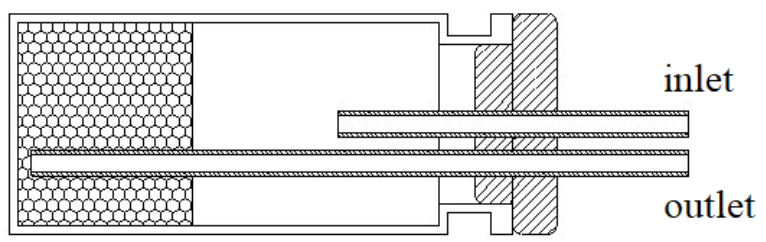

Fig. 6. Inlet / outlet for the freeze dried material in a vial

To evaluate the possibility of comparing the permeabilities of lyophilized products in vials we used two different aqueous concentrations of mannitol, which result in an utterly different porosities after freeze drying. Our previous research [14] indicates that higher concentrations of mannitol (or lactose) prolong the drying times during the same lyophilization protocol. Therefore, the higher $12 \mathrm{wt} \%$ concentration of mannitol should have a lower permeability compared to the lower $5 \mathrm{wt} \%$ concentration. Fig. 7 depicts the normalized pressure signal measured at the inlet of the vial filled with the freeze dried material.

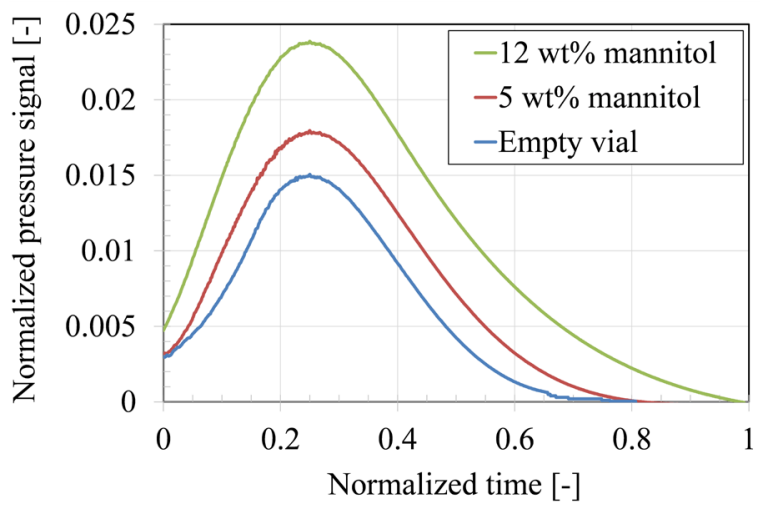

Fig. 7. Normalized pressure signal of the vials with freeze dried materials

The upstream chamber initial pressure was 1 bar during all three measurements and therefore only the material inside the vial affected the pressure signal. The results show the normalized pressure was the highest during the permeation of air through the freeze dried $12 \mathrm{wt} \%$ mannitol, which indicates that higher concentration of mannitol has a lower permeability as anticipated. The pressure rise in the empty vial occurs due to the pressure drop of the air flowing through the inlet and outlet tubing, and is therefore not in correlation with any permeability. Consequently we have made a relative comparison to the measurement of the empty vial by using the relative value of the $P T_{\text {norm, rel }}$ parameter. The derivation is in accordance 
with Eq. (5) and is more clearly presented in Eq. (8) bellow.

$$
\begin{aligned}
& P T_{\text {norm, rel }}(\text { empty })=\frac{P T_{\text {norm }}(\text { empty })}{P T_{\text {norm }}(\text { empty })}=1, \\
& P T_{\text {norm }, \text { rel }}(5 w t \%)=\frac{P T_{\text {norm }}(5 w t \%)}{P T_{\text {norm }}(\text { empty })}=1.34, \\
& P T_{\text {norm }, \text { rel }}(12 w t \%)=\frac{P T_{\text {norm }}(12 w t \%)}{P T_{\text {norm }}(\text { empty })}=2.11 .
\end{aligned}
$$

At this moment it is not yet possible to translate the $P T_{\text {norm }}$ parameter to actual permeabilities, as we have to perform the underlying measurements of a reference material in a vial. The reference material must have a known permeability and must also be placed in a vial with the same inlet/outlet arrangement to allow a straightforward derivation of the actual permeability of the lyophilized products.

\section{CONCLUSIONS}

The presented novel unsteady state method of measuring permeability was successfully evaluated with referential standardized steady state measurements performed on five different fabric materials. The presented experimental setup is capable of acquiring the entire pressure pulse signal, and the comparability parameter (slope of increasing/ decreasing pressure, pressure peak, integral parameter, or a combination of the above) is best defined after the preliminary measurements, as it depends on the properties of the measured samples. The characteristics of the fabric materials imposed the use of the integral parameter $d x / P T_{\text {norm }}$ for the comparative analysis. The validation of the measurement method yielded a linear fit curve with the $R^{2}$ of 0.98 . In the range of experimental conditions, the derived linear regression equation can be used to assess the materials' permeability measured solely with the unsteady state method. This result is extremely encouraging as only one unsteady state pressure measurement of each fabric material is included in the analysis. Moreover, the comparative analysis is based on only 141 individual pressure measurement acquired at a frequency of $4000 \mathrm{~Hz}$, which correspond to the measurement time of $35 \mathrm{~ms}$. A rapid assessment of the permeability enables possible in-line applications for quality control or similar.

The experimental results of the freezedried materials in vials have demonstrated that the presented method is capable of detecting the differences in permeabilities of lyophilized $5 \mathrm{wt} \%$ and $12 \mathrm{wt} \%$ mannitol aqueous solutions. The results show a significant change in the comparison parameter $P T_{\text {norm,rel }}$, which was 1.34 and 2.11 for the $5 \mathrm{wt} \%$ mannitol and $12 \mathrm{wt} \%$ mannitol. The parameter $P T_{\text {norm,rel }}$ increases with increasing concentration of mannitol. The higher concentrations of mannitol have smaller pores and lower permeabilities after freeze drying. Therefore the comparative parameter $P T_{\text {norm,rel }}$ increases with the decreasing permeability of the measured material, which was expected.

Several improvements of the presented novel method of measuring permeability could be implemented without any hindrance: (i) optimization of experimental equipment and operating conditions; (ii) larger number of analyzed experimental results; (iii) conditioning of the measured signal. Therefore, the future work will include: (i) measurements with reference material in a vial (ii) inclusion of multiple unsteady measurements to improve the accuracy; (iii) analysis of effects in variations of experimental setup and boundary conditions, and (iv) the use of the developed method in several additional fields and potential materials: composite materials, filters, membranes, geological samples, freeze-dried materials, 3D printed materials, artificial bones scaffold, etc. All of these applications would benefit from the analysis of the fluid permeability rate of the material under consideration. The novelty of the presented method lies in: (i) the possibility of measuring samples of arbitrary shape, nonextractable and brittle materials, (ii) the analysis of the entire pressure signal response and not just the decay during the permeation of the working fluid, (iii) the comparative analysis of the experimental results, which requires less thermodynamic properties compared to the physical modeling approach.

\section{ACKNOWLEDGEMENTS}

The author acknowledges the Department of Textiles, Graphic Arts and Design at the Faculty of Natural Sciences and Engineering, University of Ljubljana and their head prof. Barbara Simončič for the referential steady state measurements of the fabric materials' air permeability.

\section{REFERENCES}

[1] Gao, H., Li, H.A. (2016). Pore structure characterization, permeability evaluation and enhanced gas recovery techniques of tight gas sandstones. Journal of Natural Gas 
Science and Engineering, vol. 28, p. 536-547, D0l:10.1016/j. jngse.2015.12.018.

[2] Brace, W.F., Walsh, J.B., Frangos, W.T. (1968). Permeability of granite under high pressure. Journal of Geophysical Research, vol. 73, no. 6, p. 2225-2236, D0l:10.1029/JB073i006p02225.

[3] Jones, S.C. (1997). A technique for faster pulse-decay permeability measurements in tight rocks. SPE Formation Evaluation, vol. 12, no. 1, p. 19-25, D0l:10.2118/28450-PA.

[4] Dicker, A.I., Smits, R.M., (1988). A practical approach for determining permeability from laboratory pressure-pulse decay measurements. International Meeting on Petroleum Engineering, p. 8, Dol:10.2118/17578-MS.

[5] Cao, C., Li, T., Shi, J., Zhang, L., Fu, S., Wang, B., Wang, H. (2016). A new approach for measuring the permeability of shale featuring adsorption and ultra-low permeability. Journal of Natural Gas Science and Engineering, vol. 30, p. 548-556, D0l:10.1016/j.jngse.2016.02.015.

[6] Zhong, W., Tao, G., Li, X., Kawashima, K., Kagawa, T. (2011). Determination of flow rate characteristics of porous media using charge method. Flow Measurement and Instrumentation, vol. 22, no. 3, p. 201-207, D0l:10.1016/j. flowmeasinst.2011.02.002.

[7] Song, W., Yao, J., Ma, J., Li, Y., Han, W. (2018). A pore structure based real gas transport model to determine gas permeability in nanoporous shale. International Journal of Heat and Mass Transfer, vol. 126, p. 151-160, D0l:10.1016/j. ijheatmasstransfer.2018.05.012.

[8] Heidari Sureshjani, M., Ahmadi, M., Fahimpour, J. (2019). A generalized approach for fast estimation of reservoir permeability distribution from analysis of pressure transient data. Journal of Petroleum Science and Engineering, vol. 182, art. ID 106240, D0I:10.1016/j.petrol.2019.106240.

[9] Ghanizadeh, A., Gasparik, M., Amann-Hildenbrand, A., Gensterblum, Y., Krooss, B.M. (2014). Experimental study of fluid transport processes in the matrix system of the European organic-rich shales: I. Scandinavian Alum Shale. Marine and Petroleum Geology, vol. 51, p. 79-99, D0l:10.1016/j. marpetgeo.2013.10.013.

[10] Yong, A.X.H., Aktas A., May, D., ..., Willenbacher, B. (2021). Outof-plane permeability measurement for reinforcement textiles: A benchmark exercise. Composites Part A: Applied Science and Manufacturing, vol. 148, art. ID 106480, D0l:10.1016/j. compositesa.2021.106480.

[11] Swery, E.E., Allen, T., Comas-Cardona, S., Govignon, Q., Hickey, C., Timms, J., Tournier, L., Walbran, A., Kelly, P., Bickerton, S. (2016). Efficient experimental characterisation of the permeability of fibrous textiles. Journal of Composite Materials, vol. 50, no. 28, p. 4023-4038, DOI:10.1177/0021998316630801.

[12] ASTM D737-18 (2015). Standard Test Method for Determining Gas Permeability Characteristics of Plastic Film and Sheeting. ASTM International, West Conshohocken.
[13] ASTM D737-18 (2018). Standard Test Method for Air Permeability of Textile Fabrics. ASTM International, West Conshohocken.

[14] Sitar, A., Škrlec, K., Voglar, J., Avanzo, M., Kočevar, K., Cegnar, M., Irman, Š., Ravnik, J., Hriberšek, M., Golobič, I. (2017). Effects of controlled nucleation on freeze-drying lactose and mannitol aqueous solutions. Drying Technology, vol. 36, no. 10, p. 1263-1272, D0l:10.1080/07373937.2017.1399903.

[15] Arsiccio, A., Sparavigna, A.C., Pisano, R., Barresi, A.A. (2019). Measuring and predicting pore size distribution of freezedried solutions. Drying Technology, vol. 37, no. 4, p. 435-447, DOI:10.1080/07373937.2018.1430042.

[16] Metzger, T. (2019). A personal view on pore network models in drying technology. Drying Technology, vol. 37, no. 5, p. 497512, D0I:10.1080/07373937.2018.1512502.

[17] Zhou, N., Matsumoto, T., Hosokawa, T., Suekane, T. (2010). Pore-scale visualization of gas trapping in porous media by X-ray CT scanning. Flow Measurement and Instrumentation, vol. 21, no. 3, p. 262-267, D0l:10.1016/j. flowmeasinst.2010.05.002.

[18] Siebert, T., Zuber, M., Hamann, E., Baumbach, T., Karbstein, H.P., Gaukel, V. (2020). Micro-CT visualization of structure development during freeze-drying processes. Drying Technology, vol. 38, no. 3, p. 376-384, D0l:10.1080/073739 37.2019.1572619.

[19] Caglar, B., Orgeas, L., Du Roscoat, S.R., Sozer, E.M., Michaud, V. (2017). Permeability of textile fabrics with spherical inclusions. Composites Part A: Applied Science And Manufacturing, vol. 99, p. 1-14, D0l:10.1016/j.compositesa.2017.03.031.

[20] Zakirov, T., Galeev, A. (2019). Absolute permeability calculations in micro-computed tomography models of sandstones by Navier-Stokes and lattice Boltzmann equations. International Journal of Heat and Mass Transfer, vol. 129, p. 415-426, D0l:10.1016/j.ijheatmasstransfer.2018.09.119.

[21] Puszkarz, A.K., Krucińska, I. (2018). Modeling of air permeability of knitted fabric using the computational fluid dynamics. Autex Research Journal, vol. 18, no. 4, p. 364-376, DOI:10.1515/aut-2018-0007.

[22] Zhu, G., Kremenakova, D., Wang, Y., Militky, J., Mishra, R. (2015). Study on air permeability and thermal resistance of textiles under heat convection. Textile Research Journal, vol. 85, no. 16, p. 1681-1690, Dol:10.1177/0040517515573407.

[23] Ngo, N.D., Tamma, K.K. (2001). Microscale permeability predictions of porous fibrous media. International Journal of Heat and Mass Transfer, vol. 44, no. 16, p. 3135-3145, DOI:10.1016/S0017-9310(00)00335-5.

[24] Fissore, D., Pisano, R., Barresi, A.A. (2018). Process analytical technology for monitoring pharmaceuticals freeze-drying - A comprehensive review. Drying Technology, vol. 36, no. 15, p. 1839-1865, D0I:10.1080/07373937.2018.1440590.

[25] Bobba, S., Harguindeguy, M., Colucci, D., Fissore, D. (2020). Diffuse interface model of the freeze-drying process of individually frozen products. Drying Technology, vol. 38, no. 5-6, p. 758-774, DOl:10.1080/07373937.2019.1710711. 